\title{
A Study of the Influence of Path Loss and Short Term Fading on the Performance of Mobile Radio System
}

\author{
J.A.Shrawankar \\ Assistant Professor, Dept. of Electronics Engg. \\ Shri Ramdeobaba College of Engg. \& \\ Management Nagpur, India
}

\author{
K.D.Kulat, $\mathrm{PhD}$ \\ Professor \& Head, Dept. of Electronics Engg. \\ Visvesvaraya National Institute of Technology, \\ Nagpur, India
}

\begin{abstract}
The mobile radio channel places strong limitations on the performance of wireless communication systems because the transmission principle in wireless communication is more complex than those of the wired networks. In this paper we discuss the radio propagation with an objective to provide an overview of various characteristics of radio channel and an understanding of the process and factors that influences these characteristics. Section I describe a typical outdoor scenario for terrestrial mobile radio channel and principle causes of information loss (multipath fading). Section II presents the various radio channel characteristics like path loss which is used to denote the local average received signal power relative to the transmit power and helps in providing the information on coverage area. Other higher order statistical characteristics such as level crossing rate (lcr) and average duration of fade (adf), which relates the time rate of change of the received signal to the signal level and velocity of the mobile, are also considered to study the influence of short term fading on the performance of the wireless system. Section III contains the observed path loss and its comparison with the simulated path loss propagation models. Lcr and adf are obtained from the received signal envelop in section IV to study the system performance. The simulated results obtained in section III and section IV provide valuable information on coverage area, and choice of suitable data rate, word length, modulation, and coding schemes as stated in table1.
\end{abstract}

\section{General Terms}

Radio wave propagation, Mobile communication.

\section{Keywords}

Channel characterization, path loss, fading effect, mobile cellular communication, and wireless system.

\section{INTRODUCTION}

The commercial success of cellular mobile radio has led to an intense interest among wireless engineers in understanding and predicting the radio propagation characteristics for outdoor and indoor wireless applications. In modern systems, the radio links are about 20 kilometers or less, the antennas that create the link lie near to or among the buildings or even inside the buildings and the wavelength is small compared to the building dimensions. As a result, the channel characteristics are strongly influenced by the buildings as well as by vegetation and terrain. In this environment, signals propagate from one antenna to the other over multiple paths that involve the process of reflection and transmission at walls and by the ground and process of diffraction at building edges and terrain obstacles as shown in figure 1. The multipath nature of radio wave propagation as described by the various authors [1],[2]\&[3], makes itself felt in a variety of ways that have challenged the inventiveness of communication engineers. Each new concept for dealing with multipath, calls for an even deeper understanding of the characteristics of the radio channel.
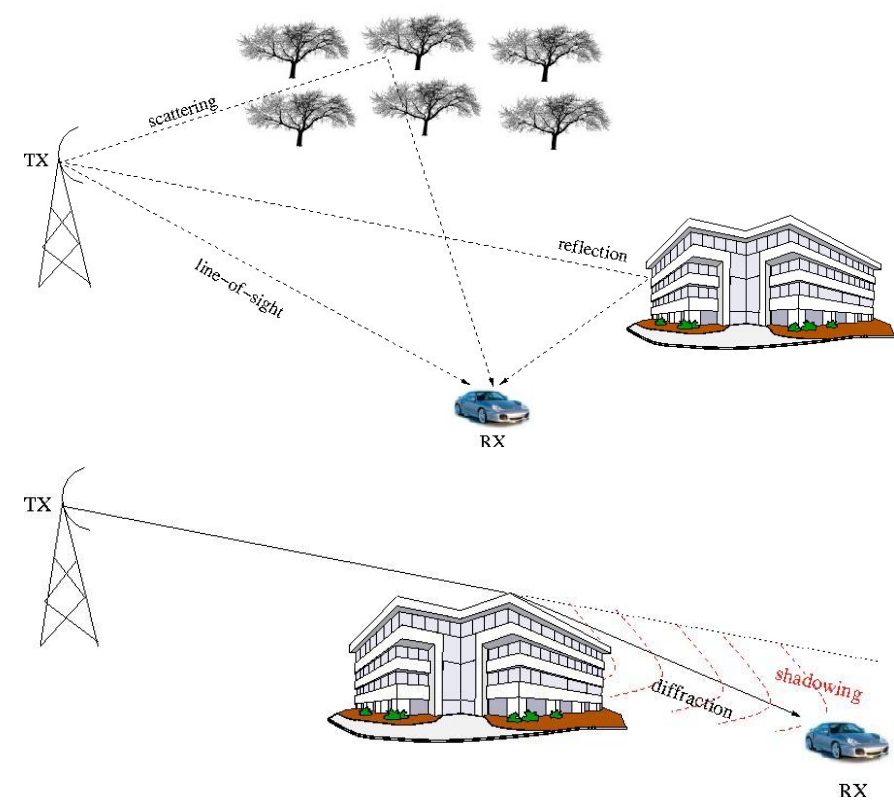

Fig. 1 A typical scenario of mobile radio communications

The mobile radio channel places strong limitations on the performance of wireless communication systems because the transmission principle in wireless communication is more complex than those of the wired networks. Unlike wired channels, whose characteristics are stationary and predictable, radio channel performance is extremely variable and needs complex analysis. Propagation models are the base for channel characterization, as they try to describe the way the radio signal changes during its travel from the transmitter to the receiver. Propagation models are useful in estimating the radio coverage area of a transmitter and the fading models characterize the rapid fluctuation of the received signal strength over a very short travel distance (few wavelengths). As the explosive growth of mobile communication continues, it is very valuable to have the capabilities of determining optimum base station locations, obtaining suitable data rates, and estimating their coverage, without conducting a series of propagation measurements which are very expensive and time consuming. 


\section{PROPAGATION CHARACTERISTICS}

\subsection{Channel Characterization based on Path loss:}

Signal propagation in wireless environment is characterized by path loss, which is the attenuation that the signal suffers from the transmitter to the receiver. A link budget calculation requires an estimate of the power level so that a signal tonoise ratio (SNR) or, similarly, a carrier-to-interference (C/I) ratio may be computed.

Path loss (PL) is used to denote the local average received signal power relative to the transmit power. This is useful quantity, since received power is usually measured as a local spatial average rather than instantaneous value. A general PL model that has been demonstrated through measurements uses a parameter called path loss exponent (n), to denote the power law relationship between distance and received power. As a function of distance, d, PL (in decibels) is expressed as [1]

$\operatorname{PL}(d)=\operatorname{PL}\left(\mathrm{d}_{\mathrm{o}}\right)+10 \mathrm{n} \log \left(\mathrm{d} / \mathrm{d}_{\mathrm{o}}\right)$

Where $\mathrm{n}$ is path loss exponent, which typically ranges from 2 (for rural) to 4 (for urban environment).

The term PL $\left(\mathrm{d}_{\mathrm{o}}\right)$ gives PL at a known close in reference distance $d_{o}$ which is in the far field of the transmitting antenna.

\subsection{Level Crossing Rate (lcr) \& Average Duration of Fade (adf)}

System engineers are interested in a quantitative description of the rate at which fades of any depth occur and average duration of a fade below any given depth. This provides a valuable aid in selecting transmission bit rates, word lengths and coding schemes in digital radio systems and allows an assessment of system performance. The required information is provided in terms of level-crossing rate and average fade duration below a specified level [2] [3].

The level crossing rate at any specified level is defined as the expected rate at which envelope crosses that level in a positive-going (or negative going) direction. The level crossing rate is a function of the mobile speed as it is apparent from below mentioned equation.

$$
\mathrm{Lcr}=\sqrt{2 \Pi} f m \rho e^{-\rho 2}
$$

Where $f m$ maximum Doppler frequency and $\rho$ normalized threshold level. As the amplitude of signal received over such a channel also fluctuates the receiver will experience periods during which signal cannot be recovered reliably; if a certain minimum (threshold) signal level is needed for acceptable communication (receiver) performance, the received signal will experience periods of sufficient signal strength or "nonfade interval", during which the receiver can work reliably and at low bit error rate.

The average fade duration defined as the average period of time for which the received signal is below a specified level A. Average duration of fade as a function of $\rho$ and $f m$ can be expressed as

$$
\text { afd }=\frac{e^{\rho 2}-1}{\rho f m \sqrt{2 \Pi}}
$$

Since lcr $\&$ adf are functions of time related to vehicle speed, they are second order statistics.

It is of critical importance to the performance of digital mobile networks that the block length or packet duration is chosen taking into account the expected duration of fades and non-fade intervals.

If the data block length is larger than the average non-fade period, almost all blocks will experience a signal fade and a corresponding burst of bit error. This may result in an excessive packet-dropping rate; unless powerful error correction codes are used.

\section{PATH LOSS MODELS}

Field measurements were peformed in the suburban city of Nagpur and nearby rural area for GSM system at $900 \mathrm{MHz}$. The measurement system as described by [6] and [7] is used for the measurement of average received signal strength at various distance away from the base station transmitter.

The measured path loss pattern is compared with the Hata, Lee and COST 231 models which are shown in figure 2, figure 3 and figure 4 respectively.

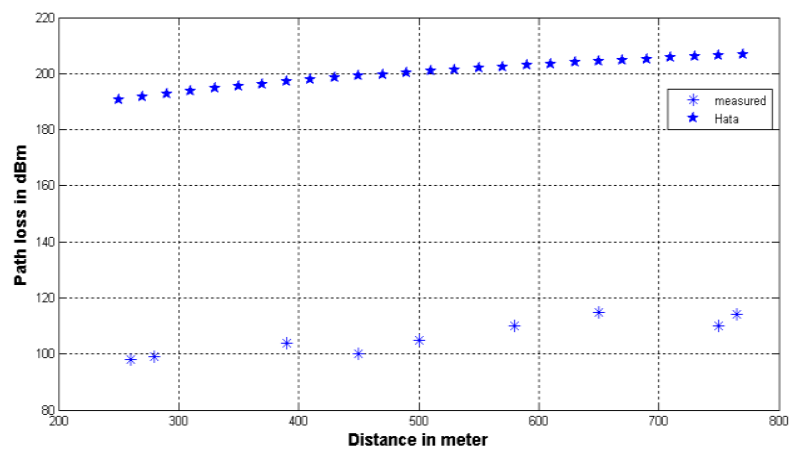

Fig.2 measured Vs Hata path loss

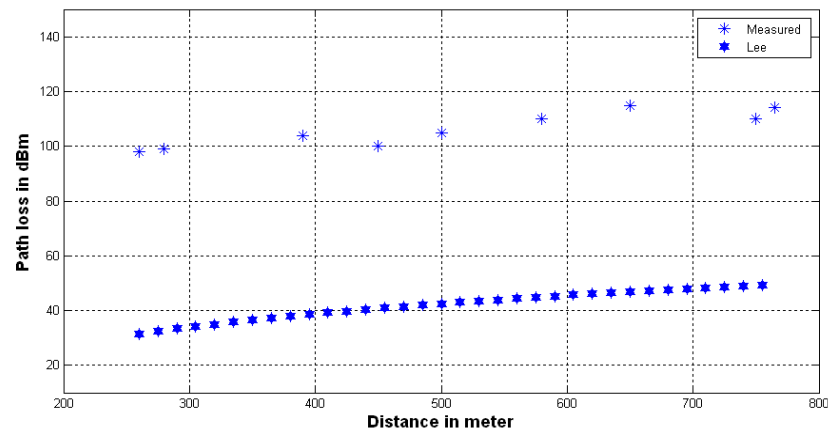

Fig.3 measured Vs Lee path loss 


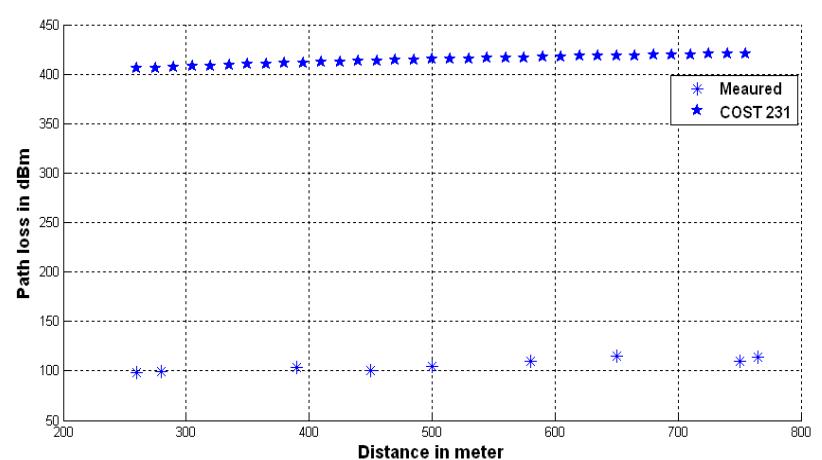

Fig.4 measured Vs COST 231 path loss

\section{CHANNEL CHARACTERIZATION}

\section{A. Simulation of fading Models based on lcr and adf [5]}

To observe the nature of received signal envelop the rural scenario is considered. As discussed in [8] [9] , and [10] the signal strength received at the mobile receiver is a random process which varies lognormaly with the distance travelled by the mobile receiver away from the transmitter.

figure 5 shown below is the Matlab simulation of received signal strength variation as a function of distance.

Simulated second order characteristic level crossing rate (lcr) $\&$ average duration of fade (adf) which depends on time is shown in figure 6 and figure 7 respectively. This provides a valuable aid in selecting transmission bit rates, word lengths and coding schemes in mobile systems and allows an assessment of system performance.

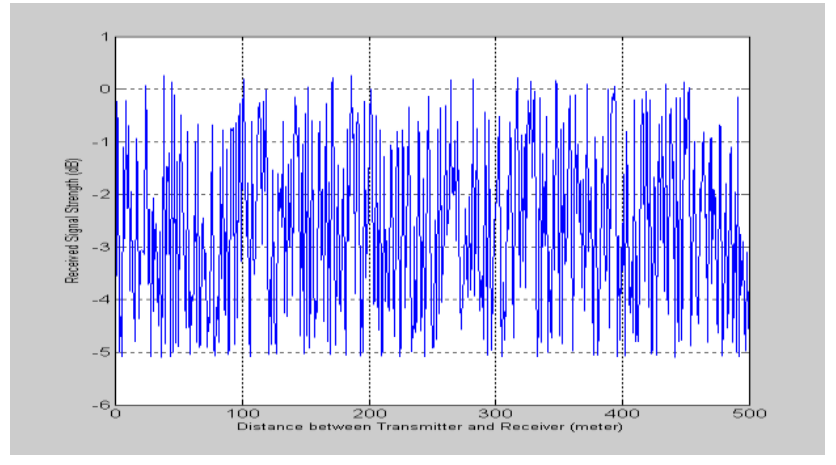

Fig.5 Received signal strength envelop

Lcr and adf is calculated using equation 2 and 3 at different Doppler frequency which is a function of vehicle speed.

For a data rate of $50 \mathrm{bps}$, the bit period is $20 \mathrm{~ms}$. The average fade duration for $\rho=1.0$ is calculated as $32 \mathrm{~ms}$. This is greater than the duration of one bit. Therefore two successive bits on average will be lost during a fade. For $\rho=1.0$, number of level crossings are calculated as 19.19 crossings per seconds. Since a bit error is assumed to occur whenever a portion of a bit encounters a fade, and since average fade duration spans over successive two bits, the total number of bits in error is $2 * 19.19=40$, resulting in a $B E R=(40 / 50)=0.8$ as indicated in table I.

It can be seen that as the vehicle speed increases, number of crossings per seconds also increases which causes more bit loss.

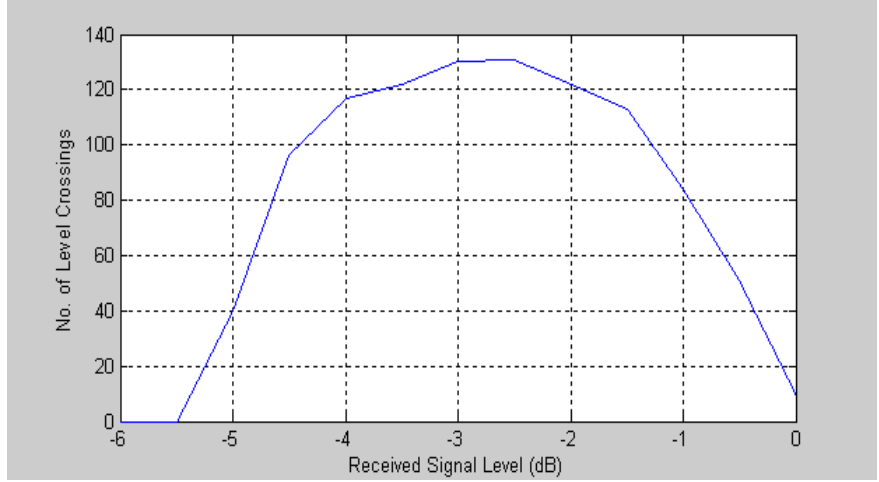

Fig. 6 Level crossing rate of received signal envelop

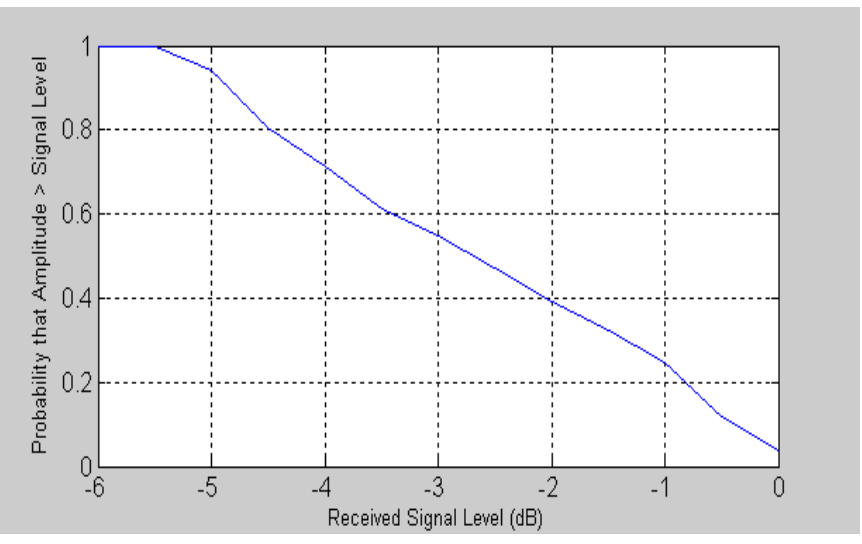

Fig.7 Average duration of fade of received signal envelop

Table 1. Simulation results for specified rms signal level

\begin{tabular}{|c|c|c|c|}
\hline $\begin{array}{c}\text { Vehicle } \\
\text { Speed } \\
(\mathbf{k m} / \mathbf{h r})\end{array}$ & lcr & adf & $\begin{array}{c}\text { BER for 50 } \\
\text { bps data } \\
\text { rate }\end{array}$ \\
\hline 25 & 19.19 & 32 & 0.80 \\
\hline 60 & 46.09 & 13 & 0.92 \\
\hline 100 & 76.83 & 8.22 & $>0.98$ \\
\hline
\end{tabular}

\section{CONCLUSION}

In this article, path loss model based on the field measurements for suburban city of Nagpur is presented. The figure 24 shows the comparison of measured data with Hata, Lee and COST models which are generally used for path loss prediction in GSM based system applicable to given terrain scenario. A comparison of the developed model with above cited models gives large difference because of different geographical conditions in India. Therefore for accurate path loss prediction, field measurements must be performed and the measured data can be used to correct the existing model or to develop a new model [8] and [10]. The simulated results indicated in Table 1 shows that bit error rate BER increases with the vehicle speed. This suggests the necessity of suitable coding scheme in order to improve the system performance. 


\section{REFERNCES}

[1] T. S. Rappaport , “Wireless communications : principles and practice" $\mathrm{PHI}$ publication,2003.

[2] Lee William C.Y., “ Mobile Communications: Design Fundamentals" John Wiley \& sons 1993

[3] Parson J.D., “ The mobile radio propagation channel “ Pentech press, 1994

[4] Sarkar T.K. et. al. " A survey of various propagation models for mobile communication" IEEE Antenna and Propagation Magazine, Vol. 45, No.3, June 2003, pp 51-82.

[5] M.F.Iskander and Z.Yun, "Propagation Prediction Models for Wireless Communication Systems," IEEE Transaction on Microwave Theory and Techniques , MTT-50, 3, 2002, pp. 662-673.

[6] Andersen J.B. , Rappaport T.S., Susumu Yoshida ," Propagation measurements and models for wireless communication channels" ,IEEE Communication Magazine Jan. 1995 , pp 42-49.
[7] J.A.Shrawankar \& K.D.Kulat "Path Loss Propagation Model for Rural and Semiurban Mobile Environment" International Journal of Engineering Research and Applications, Special issue VINCET-March 2012, pp 358-360

[8] S.Ichitsubo,T.Furuno,T.Taga,and R.Rawasaki, "Multipath Propagation Model for line-of-Sight Street Microcells in Urban Area," IEEE Transaction on Vehicular Technology ,VT-49,2,2000,pp. 422-427

[9] K.Yip and T. Ng, “ A Simulation Model for Nakagani m Fading Channels, $\mathrm{m}>1$ ", IEEE Transaction on Communications, COM-48, 2, February 2000, pp. 214 221.

[10] Gulzaib Rafiq ,Matthias Patzold “ A study of the Influence of Shadowing on the Statistical Properties of the Capacity of Mobile Radio Channel" Wireless Personal Communication :An Intenational Journal ,Vol.50, issue 1,july 2009,pp 5-18 